\title{
Color Reproduction of Metallic-Ink Images
}

\author{
Vahid Babaei and Roger D. Hersch \\ School of Computer and Communication Sciences, École Polytechnique Fédérale de Lausanne (EPFL), Switzerland \\ E-mail: babaei.v@gmail.com
}

\begin{abstract}
We study the color reproduction of full-color metallic-ink images. Full-color metallic-ink images are prints whose contributing colorants are exclusively made of colored metallic inks. Due to the presence of metallic particles, metallic inks show a metal-like luster. These particles are opaque and hide the underlying ink or substrate. In order to obtain predictable halftone colors, we need a juxtaposed halftoning method to create halftone dots of different colors side by side without overlapping. Juxtaposed halftoning invalidates many assumptions generally made for the color-reproduction workflow. For printing metallic-ink images, one needs a color-separation system creating surface coverages for the eight metallic inks that correspond to the eight Neugebauer primaries. For this purpose, we introduce a simple and fast method for $N$-color separation that relies either on Demichel's or on a variant of Kueppers' ink-to-colorant separations. Thanks to a unique set of ink-to-colorant formulas, pseudo-CMY ink values are separated into amounts of printable colorants. We also describe color-separation procedures that are able to optimize different properties of the resulting metallic-ink images. $\quad$ (C) 2016 Society for Imaging Science and Technology.

[DOI: 10.2352/J.ImagingSci.Technol.2016.60.3.030503]
\end{abstract}

\section{INTRODUCTION}

A common element present in all printing applications is the color-reproduction workflow. A color-reproduction workflow converts an input image into the printer's command language. ${ }^{1}$ Most existing reproduction workflows follow the steps shown in Figure 1. Briefly, input colors are first converted from a source color space, such as sRGB, into a device-independent color space such as CIELAB. ${ }^{2}$ By performing gamut mapping, ${ }^{3}$ the input colors are mapped into the colors of the usually narrower print gamut. Then, the color separation is carried out by converting the gamut-mapped printable colors into amounts of printer inks. The separated ink layers are then halftoned and printed.

We can use a spectral prediction model for color separation and gamut mapping. The forward prediction model expresses the forward characterization. It determines the printer's color response to input control values, e.g. the amount of inks to be printed. By varying the amount of inks in the forward model, we can determine the color gamut of a printer. The spectral prediction model is also required in inverse mode for the color separation in order to deduce the amounts of inks that are needed to print a specific color.

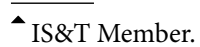

Received June 9, 2015; accepted for publication Dec. 1, 2016; published online Mar. 8, 2016. Associate Editor: Marius Pedersen.

$1062-3701 / 2016 / 60(3) / 030503 / 10 / \$ 25.00$
}

In spite of their potential applications, metallic inks have a limited usage in the printing industry. They are either used as spot colors or printed below the classic inks to give a shiny appearance to the print. In this work, we introduce full metallic-ink images, i.e. prints whose contributing colorants are exclusively made of colored metallic inks. One of the main advantages of metallic-ink prints compared to prints with classic inks on top of silver ${ }^{4}$ resides in their anti-counterfeiting capacities. Metallic-ink fulltone surfaces are not reproducible with classic inks. When embedded into color images, they are more difficult to forge. Furthermore, from a technical point of view, printing high amounts of classic inks on top of silver is not always possible because of the trapping effect. Obtaining well-saturated metallic colors with overprinting is therefore not easy.

The general color-reproduction workflow (Fig. 1) applies also for the reproduction of metallic-ink images. However, there are several challenges to be addressed. The first challenge is the halftoning of metallic inks. Since metallic inks contain opaque particles, they cannot be superposed. Classic superposition-based halftoning methods are therefore not appropriate. Juxtaposed halftoning, a perfectly dot-off-dot halftoning method, addresses this problem. ${ }^{5}$ The second challenge is the forward characterization of a metallic-ink printer. ${ }^{6}$

The third challenge for creating metallic-ink images is the color separation. The opaque particles inside the metallic inks mask the layers beneath them and prevent the formation of new colorants. Therefore, metallic-ink prints require more than four inks to provide a significant color gamut. For a gamut similar to a standard CMY gamut, eight metallic inks that correspond to the eight colorants of CMY prints are needed. But with eight inks, there will be more than one single ink combination that reproduces a desired color. This problem is also known in standard printing with custom inks and is referred to as the $N$-color-separation problem. ${ }^{7}$ The main challenge in $N$-color separation is the redundancy introduced by the additional inks.

The problem of $N$-color separation for standard prints has been studied in many works. ${ }^{8-16}$ Usually, these works divide the ink set into smaller subsets and make the color separation simpler. When there are multiple choices between different ink subsets, they might select the solution that optimizes one or more print attributes. These attributes include gamut volume, halftone visibility, color constancy, amount of consumed inks, etc. ${ }^{17}$ 


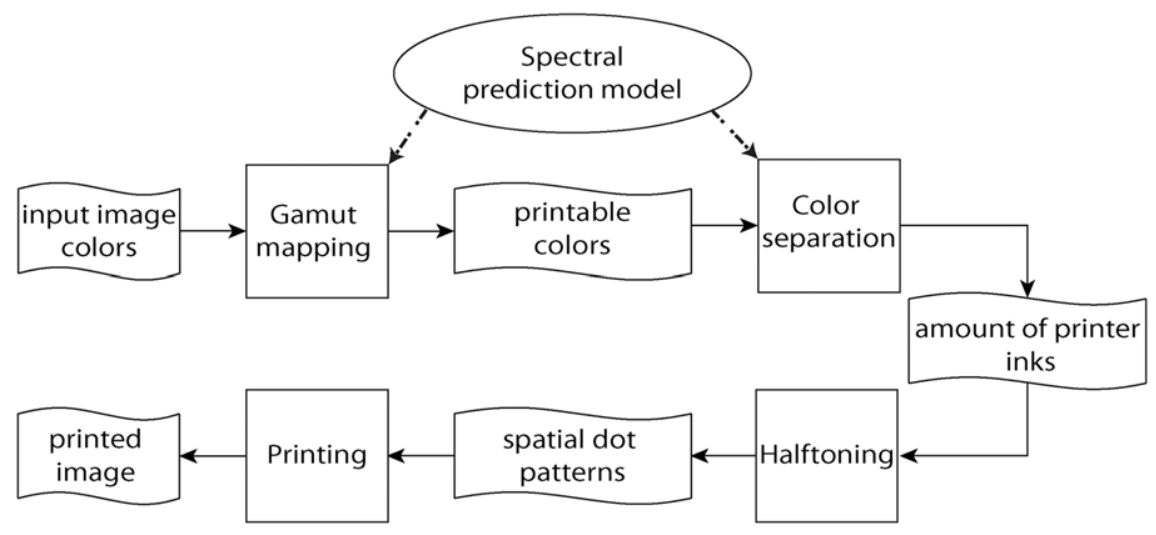

Figure 1. The color-reproduction workflow.

Chen et al. ${ }^{13}$ devise an approach toward a multi-ink color separation by using color lookup tables. For one color, they generate several ink combinations matching that color and choose the one that performs the best according to a multi-criteria print-quality metric. This metric is a weighted combination of chromaticity of the print, color constancy and graininess. However, they do not offer access to their halftoning method and no printed image is included in their results. Son et al. ${ }^{14}$ convert the CMYK digital values into CMYKLcLm ink amounts. During this conversion they take into account print-quality measures such as dot visibility and spatial smoothness of the image. Another method for color separation is halftone area Neugebauer separation (HANS). ${ }^{17}$ This method does not impose a limit on the number of contributing inks.

The present work focuses on the color separation of metallic-ink images. Inspired by gray-component replacement (GCR), ${ }^{18}$ we introduce a fast method of $N$-color separation that is both efficient and straightforward. For a given color to be separated, we first calculate an unambiguous combination of pseudo-CMY inks. The color separation is then accomplished by relying on a unique ink-to-colorant formulation that transforms obtained pseudo-CMY values into amounts of participating inks. We present two different sets of color-separation formulas and compare the print attributes resulting from the application of these formulas.

In this work, among different print attributes, we place a great emphasis on image graininess. We proceed beyond formula-based $\mathrm{N}$-color separation to improve image graininess. We show that by remembering how pixels in the neighborhood of the current pixel have been color separated, we can create smooth metallic-ink images.

\section{EXPERIMENTAL SETUP}

This section briefly explains the experimental setup that we use in our metallic-ink color-reproduction framework. We use the OKI DP-7000 printer (also known as ALPS MD) that is capable of printing with metallic inks. This desktop printer uses a thermal transfer technology for transferring the inks to the substrate. The printer can print with up to nine ink cartridges among which four are metallic: metallic cyan $(\mathrm{C})$, metallic magenta $(\mathrm{M})$, gold or metallic yellow $(\mathrm{Y})$ and silver (S).

The printer also possesses transparent process inks. In order to have a metallic-ink print with a reasonable color gamut volume, we need four more colorants. We "synthesize" three new metallic colorants: metallic blue (B) by superposing transparent cyan on metallic magenta, metallic green $(\mathrm{G})$ by superposing transparent cyan on gold and metallic red $(\mathrm{R})$ by superposing transparent magenta on gold. Finally, we add a process black ink $(\mathrm{K})$ to form a CMYKRGBS metallic-ink system. This is similar to heptatone multi-color printing ${ }^{8-10}$ except that instead of the paper we use the silver ink as "white." Because of the low registration accuracy of the printer, we use a $100 \mathrm{dpi}$ resolution.

For the halftoning, we apply the recently proposed discrete-line juxtaposed halftoning that is capable of placing any number of inks side by side without overlap. ${ }^{5}$ The halftone screen is a $1 \mathrm{D}$ superscreen with two subscreens of rational periods $T_{1}=46 / 7$ and $T_{2}=45 / 7$ with a line slope of $m=4 / 7$. Note that all eight metallic inks are juxtaposed. Figure 2 shows an example of a juxtaposed halftone screen.

As the color separation is performed by "inverting" the spectral prediction model, the forward prediction model needs to be accurate to have an accurate color reproduction. We use the nominal spectral Yule-Nielsen spectral Neugebauer model (YNSN) ${ }^{19}$ for predicting the color of metallic-ink halftones under different illumination and observation geometries. For calibration, the model needs only the eight measured fulltone Neugebauer primaries. The nominal YNSN prediction equation in vector-matrix form can be written as

$$
\mathbf{r}^{\otimes 1 / n}=\boldsymbol{A}^{\otimes 1 / n} \mathbf{a}
$$

where $\mathbf{r}$ is a 31-vector representing the halftone reflectance comprising discrete wavelengths from 400 to $700 \mathrm{~nm}$ in $10 \mathrm{~nm}$ intervals. $\boldsymbol{A}$ is the matrix of primaries whose columns are the reflectance vectors of the Neugebauer primaries $(31 \times 8)$ and $\mathbf{a}$ is the 8 -vector of nominal area coverages of these primaries. The symbol $\otimes$ stands for an element-by-element matrix operation. For classic prints, 


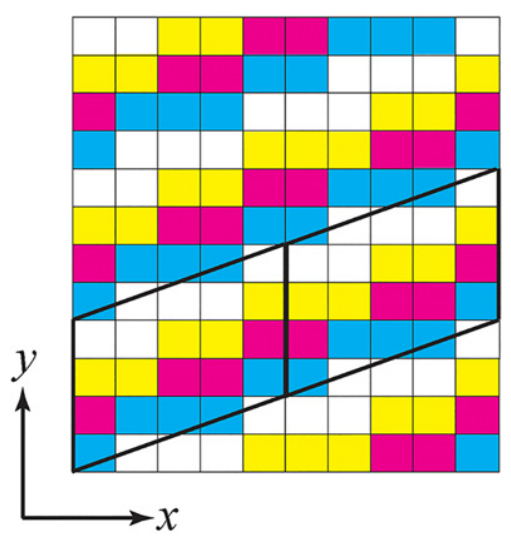

Figure 2. Discrete-line juxtaposed halftoning - a simple example of a screen comprising four colorants with different area coverages: cyan $25 \%$, magenta $20 \%$, yellow $25 \%$ and white $30 \%$. Two instances of the parallelogram screen element are shown with a solid line.

the value $n$ accounts for the optical dot gain due to lateral propagation of light inside the substrate. For metallic halftones, this parameter is mainly responsible for shadowing and misregistration between different colorants (see Ref. 6 for a thorough discussion of this effect).

In order to examine the predication accuracy of the YNSN model for metallic halftones, we consider a test set formed by 125 halftones comprising all combinations of 0 , $0.25,0.5,0.75$ and 1 nominal surface coverages of three pseudo-CMY inks from which the area coverages of the inks forming the colorants are deduced (fourth section). We use the X-Rite MA98 to perform spectrophotometric measurements at different geometries. Since the metallic halftone print is colorful under specular reflection, we view it generally at angles close to specular observation angles. For the reflectance measurements, we consider the 45 aspecular 25 geometry, i.e. $45^{\circ}$ incident angle and $20^{\circ}$ observation angle $\left(45^{\circ}: 20^{\circ}\right)$. Prints examined in front of a window are often observed according to this geometry. Light comes from the window, is reflected by the print, and a fraction of it reaches the eyes. In addition, at this geometry, the color-prediction model is accurate (mean prediction error CIE-DE2000 = 1.56). The best prediction accuracy is reached for a Yule-Nielsen $n$-value of 0.9 . For the white reference, we use the diffusing white Spectralon measured at the $45^{\circ}: 0^{\circ}$ geometry. At the $45^{\circ}: 20^{\circ}$ geometry, the silver ink is slightly darker than a diffuse white and is therefore not used as the white reference. ${ }^{20}$

\section{THE $N$-COLOR SEPARATION}

Color separation enables us to select the inks and deduce their amounts in order to print a given color. As the YNSN model is not analytically invertible, color separation is carried out by optimization techniques. ${ }^{21,22}$ Let us first formulate the color-separation problem. We search for an area-coverage vector $\mathbf{a}$ that minimizes an error metric that characterizes the distance between the predicted CIELAB color $\mathbf{c}_{p}$ and the target CIELAB color $\mathbf{c}_{t}$

$$
\mathbf{a}=\arg \min _{\mathbf{a}} \Delta E_{00}\left(\mathbf{c}_{p}(\mathbf{a}), \mathbf{c}_{\boldsymbol{t}}\right) .
$$

The CIELAB color $\boldsymbol{c}_{p}$ is calculated from the reflectance $\mathbf{r}$ that is predicted according to Eq. (1), under the D65 illuminant. In this work, we use the $\Delta E_{00}$ color-difference formula as the distance metric. $^{23}$

Simultaneous incorporation of $N$ inks in a print is challenging. First, a forward prediction model that accounts for all inks requires a significant calibration effort. Second, the total amount of ink that can be deposited on the substrate is limited. Third, the halftoning complexity increases with the number of inks. Therefore, inks are usually grouped in subsets of a small number of inks where each subset is able to reproduce a fraction of the overall gamut. This is equivalent to dividing the $\mathrm{N}$-ink gamut into multiple subgamuts. For each subgamut, we need a separately calibrated spectral prediction model.

When we are interested in a color separation with $m$ out of $N$ inks, we can iterate over all possible $m$ out of $N$ available ink subsets, i.e. $\left(\begin{array}{l}N \\ m\end{array}\right)$ combinations, and perform a color separation each time using Eq. (2). Because a color is reproducible inside more than 1 subgamut, this procedure might yield multiple answers. For the final solution, one may choose the answer that produces the lowest error. However, this is not a correct approach since the error associated with the selected answer might be only slightly lower than the error produced by other solutions. A better way is to define a set of solutions according to an error criterion, e.g., the set of solutions that satisfy $\Delta E_{00}<1$.

Juxtaposed halftoning provides us with desirable properties for simultaneously printing with a large number of inks. It lifts the constraint on the number of contributing inks for rendering a desired color. Also, as the inks are placed side by side, it resolves the issue of the total area-coverage limit. Since in this work we use the nominal YNSN model, an $N$-ink forward prediction model is straightforward to calibrate.

Having calibrated an $N$-ink color-prediction framework comprising all inks, a color separation using any number of inks can be carried out by applying the optimization formula (2) with additional constraints

$$
\begin{aligned}
& \mathbf{a}=\arg \min _{\mathbf{a}} \Delta E_{00}\left(\mathbf{c}_{p}(\mathbf{a}), \mathbf{c}_{t}\right) \\
& \text { subject to }\left\|\mathbf{a}_{q}\right\|_{1}=1 \\
& \text { and } \mathbf{a} \in[0,1]^{m}(1 \leq m \leq N)
\end{aligned}
$$

where $\|.\|_{1}$ stands for the 1 -norm of a vector, i.e. the sum of its elements. We execute multiple times the minimization (3) on all possible subgamuts of $m$ inks. Each time, the sum of ink area coverages inside the $q$ th subgamut is 1 . Consequently, all nonparticipating inks that do not belong to this subgamut have zero area coverages. For the minimization, we use the MATLAB fmincon function that computes a quasi-Newton approximation to the Hessian of the Lagrangian. 


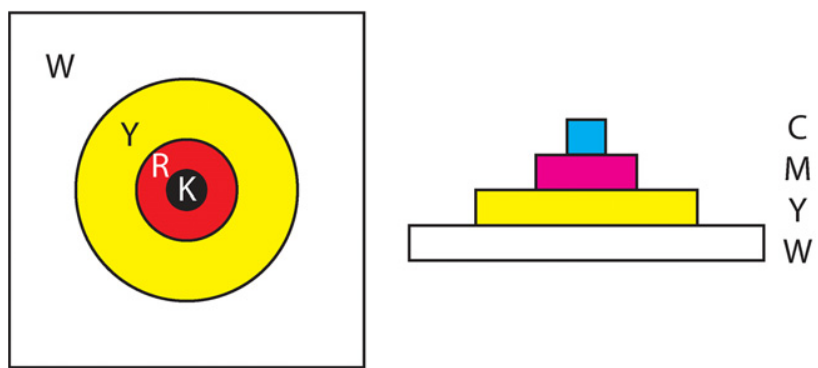

Figure 3. Schematic representation of a dot-on-dot screen, top view on the left and cross-section on the right. In this example, $c \leq m \leq y$.

\section{$N$-COLOR SEPARATION RELYING ON A SEPARATION FORMULA}

We are interested in a fast color-separation method for metallic-ink image reproduction. We are inspired by the gray-component replacement (GCR) that is used for printing with CMYK. ${ }^{18}$ In this method, first the color separation for three chromatic inks is calculated. Then, during the black generation step, the amount of black ink is obtained by a unique transform from CMY to CMYK. In a similar approach, we first accomplish a color separation for a pseudo-CMY ink system. We call it pseudo-ink system because we do not reproduce the colors with CMY inks only, but rather with the eight metallic inks (CMYKRGBS). Therefore, we consider ink-to-colorant separation formulas mapping the pseudo-CMY inks to all contributing colorants (CMYKRGBS).

For the color separation relying on a set of formulas, pseudo surface coverages of cyan, magenta and yellow are converted into area coverages of colorants using the considered ink-to-colorant mapping. The separation formulas provide a relationship that is equivalent to the division of the overall gamut into a number of subgamuts. However, the ink-to-colorant formulas accomplish this task implicitly with a mathematical formula that is computed very rapidly. Depending on the separation formulas, a given color in the gamut is assigned to a specific subgamut. As we rely on juxtaposed halftoning, the separation formulas should result in area coverages that sum up to 1 . We consider two sets of formulas for the color separation of metallic-ink prints.

\section{Kueppers' Ink-to-colorant Separation Formulas}

Kueppers $^{8}$ uses a CMYKRGB ink set to create heptatone prints. In his approach, every color in the color space is reproduced by up to four inks, two of which are black and white. In other words, in every color separation there exist at most two chromatic inks. The proposed formulation can best be described by considering pseudo-CMY transparent inks superposed as in dot-on-dot screens ${ }^{21,24}$ (see Figure 3). The outcome of the superposition depends on the respective pseudo-CMY surface coverages $(c, m, y)$. In Fig. 3, the CMY ink surface coverages have the relationship $c \leq m \leq y$; hence, according to Table $I$, the resulting area coverages of the Neugebauer primaries are $a_{y}=y-m, a_{r}=m-c, a_{k}=c$ and $a_{w}=1-y$.
Table I. The area coverages of the eight Neugebauer colorants (primaries), according to Kueppers' ink-to-colorant separation method. The area coverages $a_{c}, a_{m}, a_{y}, a_{k}$, $a_{r}, a_{g}, a_{b}, a_{W}$ of the colorants cyan, magenta, yellow, black, red, green, blue and white, respectively, depend on the corresponding $c, m, y$ surface coverages of the pseudo-CMY inks.

\begin{tabular}{lllllll}
\hline & $c \leq m \leq y$ & $c \leq y<m$ & $m<c \leq y$ & $m \leq y<c$ & $y<c \leq m$ & $y<m<c$ \\
\hline$a_{c}$ & 0 & 0 & 0 & $c-y$ & 0 & $c-m$ \\
$a_{m}$ & 0 & $m-y$ & 0 & 0 & $m-c$ & 0 \\
$a_{y}$ & $y-m$ & 0 & $y-c$ & 0 & 0 & 0 \\
$a_{k} \quad c$ & $c$ & $M$ & $m$ & $\gamma$ & $y$ \\
$a_{r}$ & $m-c$ & $y-c$ & 0 & 0 & 0 & 0 \\
$a_{g}$ & 0 & 0 & $c-m$ & $y-m$ & 0 & 0 \\
$a_{b}$ & 0 & 0 & 0 & 0 & $c-y$ & $m-y$ \\
$a_{w}$ & $1-y$ & $1-m$ & $1-y$ & $1-c$ & $1-m$ & $1-c$ \\
\hline
\end{tabular}

Table II. The surface coverages of eight Neugebauer colorants (primaries) according to Demichel's separation formulas.

\begin{tabular}{llll}
\hline$a_{c}$ & $c(1-m)(1-y)$ & $a_{r}$ & $(1-c) m y$ \\
$a_{m}$ & $(1-c) m(1-y)$ & $a_{g}$ & $c(1-m) y$ \\
$a_{y}$ & $(1-c)(1-m) y$ & $a_{b}$ & $c m(1-y)$ \\
$a_{k}$ & $c m y$ & $a_{w}$ & $(1-c)(1-m)(1-y)$ \\
\hline
\end{tabular}

Table I lists all possible colorant area coverages that result from different respective surface coverages of cyan, magenta and yellow in a dot-on-dot screen. Note that the sum of all colorant area coverages in each column is 1 . Note that the dot-on-dot approach is only used to explain Kueppers' color-separation method. As the metallic inks are opaque, the colorant areas are halftoned in a perfectly juxtaposed manner.

\section{Demichel's Ink-to-colorant Separation Formulas}

The Demichel ink-to-colorant separation formulas, also known as Demichel equations, are extensively used in halftone printing. Table II summarizes the Demichel formulas. Given the surface coverages of the pseudo-CMY inks, the Demichel formulas compute the area coverages of the eight Neugebauer primaries (colorants). They rely on the statistically independent superposition of ink dots. ${ }^{25}$ We can verify that the sum of all area coverages in the Demichel formulas is 1 . Note that using the Demichel pseudo-ink to colorant separation formulas does not imply that the colorants should be laid out independently. The separation formulas only create a one-to-one relationship between pseudo-CMY inks and the area coverages of the actual colorants. It reduces the 8-ink color-separation problem into the simpler pseudo-CMY ink color separation.

Let us compare different print characteristics of test patches color separated according to the considered separation schemes. 
Table III. The volume of the concave gamut of metallic-ink print at $45^{\circ}: 20^{\circ}$ geometry achievable with different sets of ink-to-colorant separation formulas (in kilo CIELAB).

\begin{tabular}{lll}
\hline Kueppers' formulas & Demichel's formulas & Largest gamut \\
\hline 156.02 & 148.39 & 168.78 \\
\hline
\end{tabular}

\section{Gamut Volume}

The first criterion for evaluating a set of separation formulas is the corresponding resulting gamut volume. If the gamut volume is significantly smaller than the maximum volume achievable with a given printing system, the corresponding set of separation formulas fails to cover all regions of the color gamut and is therefore not suitable for high quality color reproduction. To calculate the gamut volume for a given set of printer, paper and inks, we use the YNSN model (Eq. (1)) and predict the corresponding reflection spectra. We run this model by predicting the reflectances of every possible combination of colorant area coverages, for example in steps of $5 \%$ under the constraint that the sum of area coverages is 1. Each spectrum is then converted to CIEXYZ, and subsequently to CIELAB under the D65 illuminant. From the set of CIELAB colors synthesized using the prediction model, we perform a Delaunay tetrahedralization and compute the concave hull with the alpha-shapes technique. ${ }^{26}$ From this concave hull, the gamut volume is deduced.

We apply this methodology to calculate the gamut volume covered by the ink-to-colorant separation formulas. We predict the colors of all possible combinations of surface coverages of the three pseudo-CMY inks. They can take any surface coverage between 0 and 1 . Using either Kueppers' or Demichel's ink-to-colorant separation formulas, we compute the area coverages of the eight colorants, here the eight metallic inks. We then obtain the gamut volume corresponding to each set of formulas. Table III shows the calculated gamut volume for each separation strategy. Kueppers' and Demichel's sets of formulas cover $93 \%$ and $88 \%$ of the largest possible gamut, respectively.

Since we use the same inks and the same prediction model, the differences in gamut volume are only due to differences in the sets of separation formulas. Different sets of formulas cover different regions in the domain of colorant area coverages (metallic inks). Table IV lists the subgamuts accessible by each set of separation formulas. For the sake of completeness, we count every single ink as a subgamut. In Table IV, a subgamut named $A_{1} A_{2} \cdots A_{M}$ is formed by combinations of nonzero amounts of inks $a_{1}, a_{2} \cdots a_{M}$ where the sum of their corresponding area coverages is unity, i.e. $a_{1}+a_{2}+\cdots+a_{M}=1$.

We can verify the existence of Kueppers' subgamuts by considering superposed dot-on-dot configurations according to Fig. 3 and to the equations given in Table I. For example, the configuration showed in Fig. 3 with pseudo-CMY surface coverages $c<m<y$ yields a 4 -ink black, red, yellow and white subgamut (KRYW). With pseudo-CMY surface coverages $c<m=y$, the resulting gamut becomes a 3-ink black, red, white subgamut (KRW).
Table IV. List of all subgamuts available according to the different ink-to-colorant separation strategies. The K, R, G, B, C, M, Y, W primaries (colorants) are obtained by either Kueppers' or Demichel's separation formulas.

\begin{tabular}{|c|c|c|}
\hline Subgamut & Kueppers (52) & Demichel (27) \\
\hline l-ink & $8(K, R, G, B, C, M, Y, W)$ & $8(K, R, G, B, C, M, Y, W)$ \\
\hline \multirow[t]{3}{*}{ 2-ink } & 19 (KW, GC, BC, CW, KC, & \\
\hline & RM, BM, MW, KM, RY, GY, & 12 (GC, BC, CW, RM, BM, MW, \\
\hline & $\begin{array}{c}\text { YW, KY, RW, KR, GW, KG, } \\
\text { BW, KB) }\end{array}$ & RY, GY, YW, KR, KG, KB) \\
\hline \multirow[t]{5}{*}{ 3-ink } & 19 (KCW, BCW, KBC, KBW, & \\
\hline & KMW, BMW, KBM, RMW, & \\
\hline & RMK, KYW, RYW, KRY, & 0 \\
\hline & KRW, GYW, KGY, KGW, & \\
\hline & G(W, KGC, KGW) & \\
\hline \multirow[t]{2}{*}{ 4-ink } & 6 (KGCW, KBCW, KRMW, & 6 (BCMW, GCYW, KGBC, \\
\hline & KBMW, KRYW, KGYW) & RMYW, KRBM, KRGY) \\
\hline 5-ink & 0 & 0 \\
\hline 6-ink & 0 & 0 \\
\hline 7-ink & 0 & 0 \\
\hline 8-ink & 0 & 1 (KRGBCMYW) \\
\hline
\end{tabular}

Let us also clarify the subgamuts achievable with Demichel's formulas. With Demichel's formulas, each pseudo $\mathrm{C}, \mathrm{M}$ or $\mathrm{Y}$ ink can freely take a value in the range $[0,1]$. Each of these three inks can be equal to 0 or 1 or take a value in the interval $(0,1)$. These three possible value ranges for the three pseudo-CMY inks yield $3^{3}(=27)$ subgamuts. For example, if cyan and magenta are 0 , this results in "subgamut" $Y$. If cyan is 1 , magenta 0 and yellow is in $(0,1)$, it results in subgamut GC. If cyan is 1 and magenta and yellow are in $(0,1)$, we obtain subgamut KBGC. If the three inks are in $(0,1)$ we have an 8-ink gamut KRGBCMYW.

Note that the subgamuts in Table IV are enumerated by assuming that larger subgamuts cannot be reduced to subgamuts with a lower number of inks. The colorants in each subgamut cannot have 0 values. For example, with Demichel's formulas, a 3-ink subgamut, e.g. CMW, cannot be achieved by setting to zero the value of the blue ink in the 4-ink subgamut CMBW.

Both Kueppers' and Demichel's separation formulas are nonlinear transformations from $[0,1]^{3}$ to $[0,1]^{8}$. Each set of formulas spans a specific range in $[0,1]^{8}$. Note that, in addition to the general limitation of the sum of area coverages and to the nonzero condition, there are other limitations specific to each set of formulas. The colorants in the 8-ink gamut accessible by the Demichel formulas cannot take any arbitrary nonzero values. The constraints induced by the Demichel formulas limit the range of accessible colorant area coverages. For example, increasing the value of the cyan colorant independent of the other colorants is not possible. An increase in the value of the cyan pseudo-ink results in increased values of the blue, green and black colorants.

Table IV indicates why the Demichel separation formula offers a gamut volume smaller than Kueppers' separation 
formulas. With Demichel's formulas, we can verify that any 2-colorant combination comprising black and one colorant selected from cyan, magenta and yellow is not possible. These combinations represent colors at the border of the gamut and have a direct influence on the gamut volume. Kueppers' formulas, however, can produce these combinations of colorants.

\section{Maximum Number of Colorants (Metallic Inks) per Color}

This criterion determines the maximum number of colorants (i.e. metallic inks) that can be placed in a halftone screen element to reproduce a given color. For direct color separation, it can be set to any number. When using separation formulas, the maximum number of colorants per screen is known beforehand. Kueppers' separation formulas use at most four colorants to reproduce a color. The Demichel separation formulas need a maximum of eight colorants per color. The number of colorants per color to be reproduced has an important influence on the halftone properties. A small number of colorants in a halftone yield for each colorant a larger dot, thus leading to more clustering. This can be advantageous for some printing technologies where small dots tend to disappear. Furthermore, with larger dots, the color shift induced by the misregistration of the different layers has less undesirable effects. ${ }^{27}$

\section{Color Constancy}

The CMCCON97 Color Inconstancy Index is used to evaluate the color constancy of samples generated by different color-separation strategies. In order to compute this index for any color sample we carry out the following steps: ${ }^{28}$

(1) We measure or calculate the CIEXYZ values of the sample under a test $\left(X Y Z_{t}\right)$ and a reference illuminant $\left(X Y Z_{r}\right)$.

(2) A chromatic adaptation transform (e.g. CMCCAT97) ${ }^{28}$ is used to compute the corresponding color $X Y Z_{C A T}$ of the sample when taken from the test to the reference illuminant.

(3) The Color Inconstancy Index is defined as the color difference between the actual color $\left(X Y Z_{r}\right)$ and the transformed color of the sample $\left(X Y Z_{\mathrm{CAT}}\right)$ under the reference illuminant.

We first perform the color separation on 125 color patches printed with metallic inks by using either Kueppers' or Demichel's set of separation formulas. As these colors are inside the printer gamut, no gamut clipping occurs. Having obtained the area coverage of a halftone, we predict its spectral reflectance. Tri-stimulus values are then calculated from the predicted spectral reflectance for the test illuminant and the reference illuminant. We set fluorescent F11 (also known under the name TL84) and D65 as test and reference illuminants, respectively. The CIE-DE2000 color-difference formula is then used to evaluate the difference between pairs of corresponding colors. Table $\mathrm{V}$ shows the statistics of the CMCCON97 Color Inconstancy Index for 125 representative test colors separated according to the two sets of separation
Table V. The CMCCON97 Color Inconstancy Index of 125 metallic-ink test-halftones color separated using different separation strategies, with $\mathrm{Fll}$ as test illuminant and D65 as reference illuminant.

\begin{tabular}{lll}
\hline CII & Kueppers' formulas & Demichel's formulas \\
\hline Mean & 3.14 & 4.24 \\
0.95 quantile & 5.65 & 7.41 \\
Max & 7.24 & 9.07 \\
\hline
\end{tabular}

Table VI. The halftone visibility score (std-SL*) based on S-CIELAB for 125 test-halftones color separated using different separation schemes.

\begin{tabular}{lll}
\hline Visibility score & Kueppers' formulas & Demichel's formulas \\
\hline Mean & 12.8 & 12.3 \\
0.95 quantile & 27.5 & 24.9 \\
Standard dev. & 9.8 & 7.4 \\
\hline
\end{tabular}

formulas. The 125 colors are formed by all combinations of pseudo-CMY surface coverages of $0,0.25,0.5,0.75$ and 1 . Halftones separated using Kueppers' formulas show higher color constancy when observed under different illuminants. This is due to the presence of higher amounts of achromatic black and silver inks compared to the halftones color separated with Demichel's separation formulas.

\section{Halftone Visibility}

As the discrete-line juxtaposed halftoning tends to produce $1 \mathrm{D}$ lines that are more visible than other dot shapes, halftone visibility is particularly important. The metric we use to evaluate the halftone visibility is the standard deviation of the lightness $L^{*}$ in a constant-color spatial-CIELAB halftone image. ${ }^{14}$ We call this metric std $-S L^{*}$. In order to compute it for a halftone with a certain area-coverage vector a, we generate the halftone tile using discrete-line juxtaposed halftoning. We then create a CIEXYZ halftone image with the CIEXYZ values of the eight inks assigned to each pixel. From the XYZ halftone image we can generate the spatial-CIELAB halftone image by low-pass filtering as is done in S-CIELAB. ${ }^{29}$ We set the viewing distance to 18 inches and the image resolution to $100 \mathrm{dpi}$, which is our printer resolution. The standard deviation of the $L^{*}$ channel of the S-CIELAB halftone image is adopted as the visibility score. The higher the score, the higher the visibility of the halftone.

Table VI shows the $s t d-S L^{*}$ statistics for the 125 representative halftone patches with separated area coverages according to the different color-separation formulas. Kueppers' formulas yield slightly more visible halftones. Although the mean visibility score for both methods is almost equal, Kueppers' formulas result in a higher standard deviation of the $s t d-S L^{*}$ visibility score. This is because the visibility is high for halftone patches with large amounts of black and silver and low for halftones with less black and silver inks. We further discuss halftone visibility in the next section. 


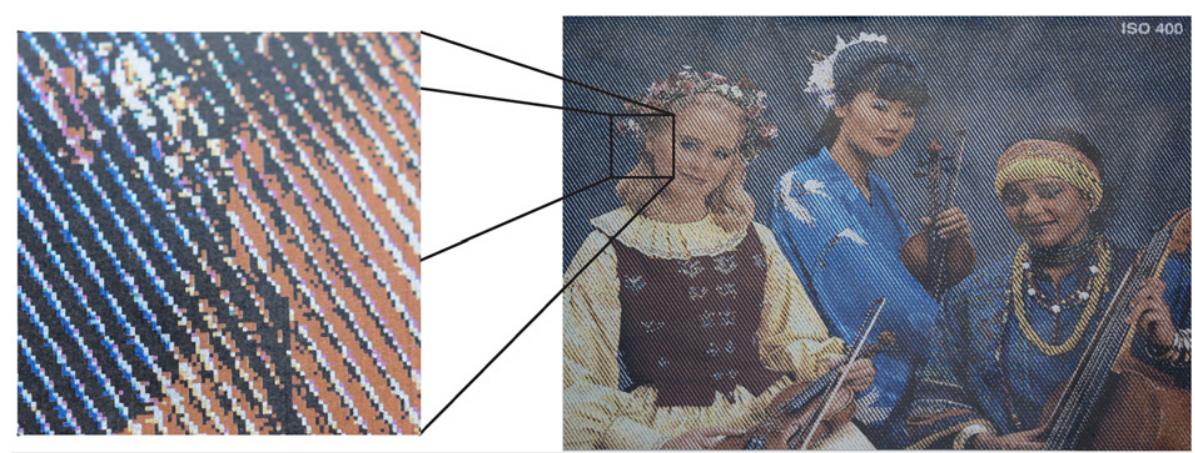

(a) Kueppers

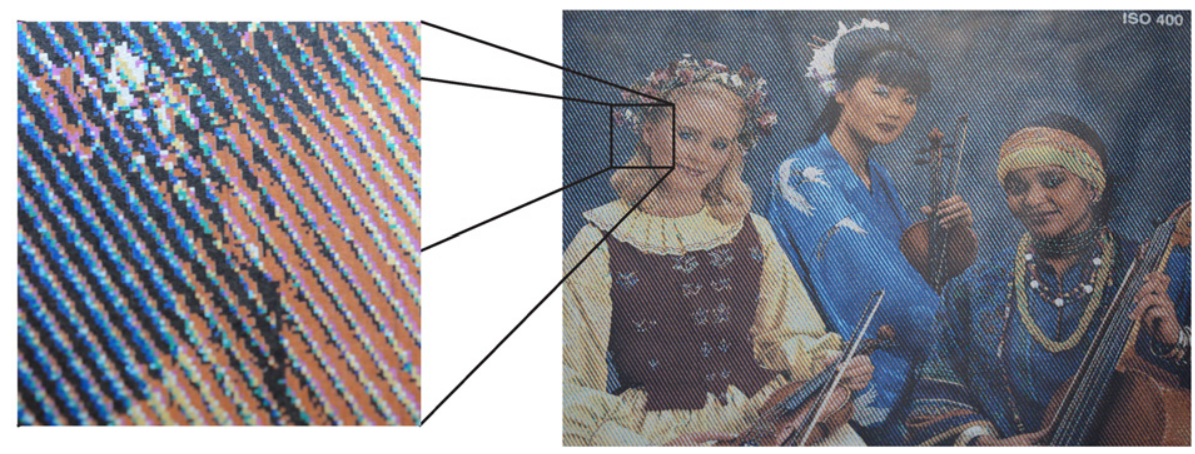

(b) Demichel

Figure 4. Photographs of two full metallic-ink prints produced by our workflow viewed under the 45 aspecular 25 geometry $\left(45^{\circ}: 20^{\circ}\right)$. The only difference between these two images is the set of separation formulas. The top image is generated by Kueppers' formulas and the bottom image with Demichel's formulas (see the electronic version).

\section{Printed Results}

Figure 4 shows the photographs of two printed images reproduced using our reproduction workflow for metallic inks. The only difference between these two images is the set of formulas used for their color separation. Both images are halftoned with a $1 \mathrm{D}$ discrete-line superscreen with slope $m=4 / 7$ and vertical period $T=45 / 7+46 / 7$. We use the YNSN prediction model with $n=0.9$ to establish the gamuts and perform the color separation. As shown in this figure, the image generated using Kueppers' formulas looks more vivid. This is due to the high-contrast screens resulting from screens having a lower number of inks and to the presence of the black ink. Its gamut is also slightly larger.

\section{$N$-COLOR SEPARATION WITH ATTRIBUTE OPTIMIZATION}

In this section, we carry out the color separation with the aim of optimizing some desired print properties. We are particularly interested in halftone visibility and image graininess. Halftone visibility and image graininess are two different attributes. Halftone visibility concerns the visibility of halftone dots when reproducing uniformly colored patches. It is an important property for reproducing spot colors with $N$ inks. ${ }^{22}$ Image graininess refers to color images and expresses the amount of "grain" in an image. Depending on the color separation, the graininess of an image can change.

\section{Optimizing Halftone Visibility}

In this section, we minimize the halftone visibility when reproducing a given color. For this purpose, we perform a color separation by considering all possible subgamuts of our 8 -ink system. Applying the binomial theorem, we can verify that there are

$$
\left(\begin{array}{c}
N \\
1
\end{array}\right)+\left(\begin{array}{l}
N \\
2
\end{array}\right)+\cdots+\left(\begin{array}{l}
N \\
N
\end{array}\right)=2^{N}-1
$$

subgamuts in an $\mathrm{N}$-ink printing system. Therefore, there are 255 subgamuts in an 8 -ink system. After choosing the candidate subgamuts capable of reproducing the desired color with high colorimetric accuracy, we can select among them the subgamut with the lowest visibility score.

A brute-force approach for visiting all 255 subgamuts is computationally expensive. In order to speed up the procedure, we first exclude the subgamuts that certainly do not contain the desired color. This can be achieved by considering the bounding boxes of all subgamuts in CIELAB. In order to separate a given color, we exclude the subgamuts that do not contain that color. Furthermore, we sort the subgamuts that satisfy the bounding box condition according to a rough visibility metric. This metric is the standard deviation of the CIELAB $L^{*}$ component of the contributing inks in each subgamut, for equal colorant area coverages.

We set the thresholds of colorimetric accuracy and halftone visibility to $\Delta E_{00}=1.5$ and $s t d-S L^{*}=10$, 


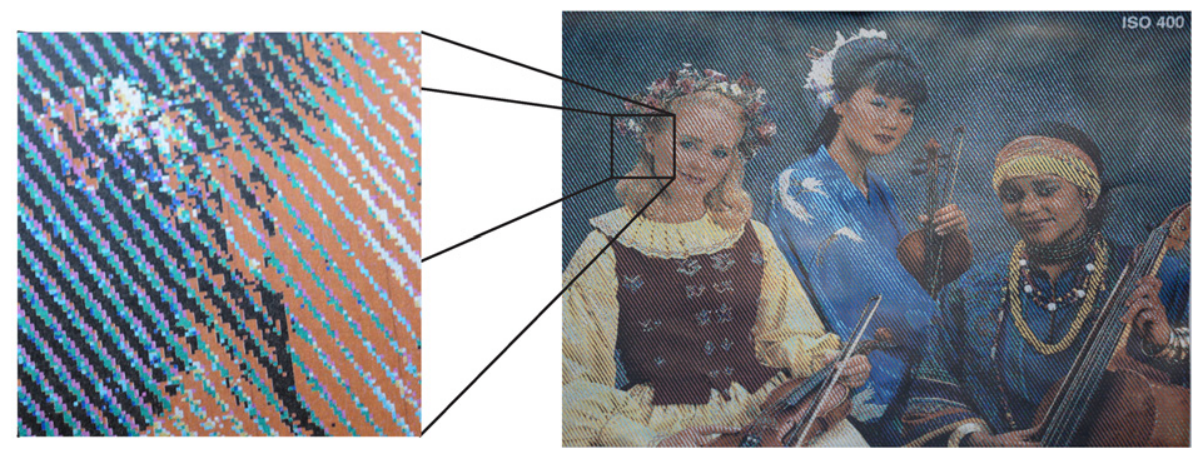

Figure 5. Photographs of a full metallic-ink print under the 45 aspecular 25 geometry with optimized halftone visibility (see the electronic version). Note that $4 \%, 28.2 \%, 44.1 \%, 20 \%$, and $3.7 \%$ of the pixels are color separated with a 2-ink, 3-ink, 4-ink, 5-ink and 6-ink subgamut, respectively.

respectively. For a given color, we search among the valid subgamuts sorted according to the rough visibility metric. Beginning with the first valid subgamut with the least $L^{*}$ deviation, we perform the color separation. If the colorimetric accuracy threshold is met, we calculate the halftone visibility score $\left(s t d-S L^{*}\right)$ of the separation solution according to the procedure described earlier. If the visibility threshold is also met, we set that solution as the final area-coverage vector. If the visibility threshold is not met after visiting all valid subgamuts, we choose among the accurate separation solutions the one with the lowest visibility score.

Note that the halftone-visibility thresholding method is suitable for minimizing the visibility of uniform halftones. This method does not necessarily yield smooth color images. Figure 5 shows the photograph of a full metallic print generated by minimizing the halftone-visibility score for each pixel. The image shows a higher level of graininess than the images produced with the separation formulas (Fig. 4). The reason is that in this scheme, we are using all possible subgamuts. Although the individual halftones are less visible (observable in uniform areas of the image), switching between different subgamuts makes the whole image look grainier. With the separation formulas, the number of subgamuts is more limited and, although the halftone visibility is higher, the image graininess is lower.

\section{Optimizing Image Graininess}

Color-separation-induced image graininess is a local property and increases with increased dissimilarity between the subgamuts that are located within a close neighborhood. For example, as can be seen in Fig. 4, the image generated by Demichel's separation formulas tends to be smoother. According to Table IV, the number of subgamuts accessible with Kueppers' formulas (52 subgamuts) is larger than with Demichel's formulas (27 subgamuts). Also, the higher standard deviation in the visibility of screens generated by Kueppers' formulas leads to a grainier image.

In order to limit the number of accessible subgamuts within a small area in the image, when performing pixel by pixel color separation, we give the preference to the

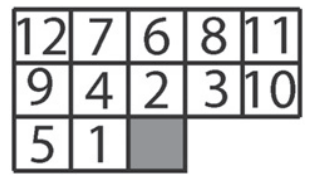

Figure 6. Priority of visited subgamuts during color separation of a small region of an image. The subgamuts used for color separation of the gray pixel are sorted according to their vicinity to this pixel.

subgamuts visited by adjacent pixels. Inspired by the Stucki weights for error-diffusion halftoning, ${ }^{30}$ we set the preferences using the order shown in Figure 6. To perform the color separation for the current pixel (shown by the gray square in the Fig. 6), we use the subgamut with which adjacent pixel number 1 was color separated. If the threshold accuracy is met, we proceed to the next pixel. Otherwise, we try other subgamuts in the specified order. If none of the 12 subgamuts give a satisfactory color separation, we test all remaining subgamuts sorted by their rough visibility metric.

Figure 7 shows the same image color separated by neighborhood-subgamut processing. In this example, we use only 5-ink subgamuts and set the accuracy threshold to $1.5 \Delta E_{00}$ color difference. The generated image is visibly smoother than all other color separated metallic-ink prints shown in this article.

Graininess in smooth image regions is partly induced by false boundaries resulting from changes in subgamuts, especially under an illuminant which differs from the illuminant used for the color separation. We therefore evaluate the changes in subgamuts within small regions of the analyzed image. For this purpose, we estimate the similarity between visited subgamuts of neighboring regions. We first assign to each pixel in the halftone image the index of its subgamut. Then, we build histogram vectors representing the subgamut indexes and their number of occurrences in small regions of the halftone image $(10 \times 10$ pixels for example). The similarity metric is defined as the cosine of the angle between the histogram vectors of neighboring pixel blocks. Averaging this metric over the whole image gives 0.82 for the print shown in Fig. 7 and 0.72 for the print shown in Fig. 5. The print in Fig. 7 has therefore less changes in subgamuts 


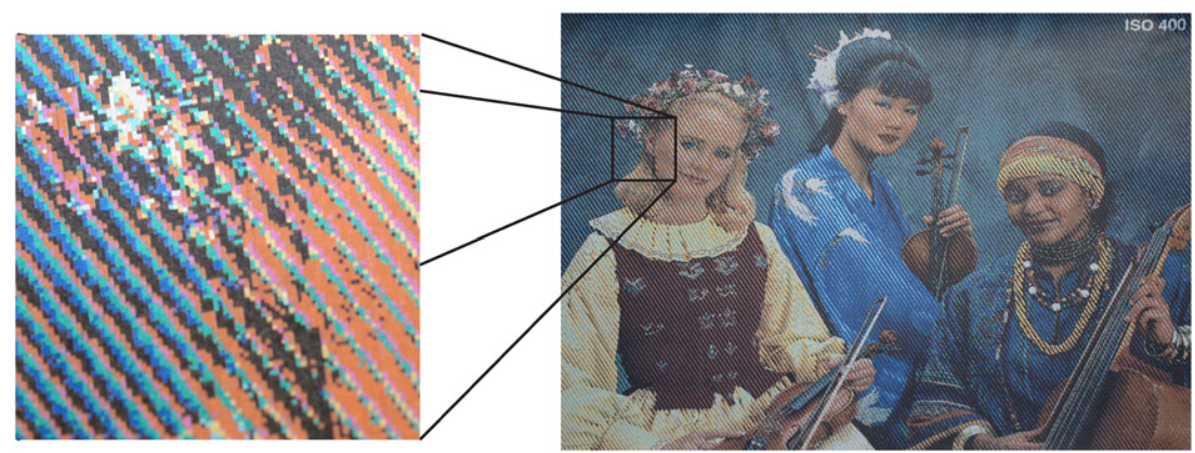

Figure 7. Photographs of a full metallic-ink print under the 45 aspecular 25 geometry with neighborhood-subgamut processing (see the electronic version). Note that $0.9 \%, 15.1 \%, 35.5 \%, 48.5 \%$ of the pixels are color separated with a 2-ink, 3-ink, 4-ink, and 5-ink subgamut, respectively.

in neighboring regions compared with the print of Fig. 5. Its potential for graininess is therefore lower, especially under illuminants which differ from the D65 illuminant used by the color-prediction model for the color separation.

\section{CONCLUSIONS}

Although the main purpose of color separation is accuracy in printing the desired color, there are other print attributes that depend on the color-separation method. We addressed the problem of color separation of juxtaposed metallicink halftones. $\mathrm{N}$-color printing is necessary for printing metallic-ink images. Due to the flexibility of the discrete-line juxtaposed halftoning enabling to print simultaneously with a large number of inks, we can benefit from inkto-colorant separation formulas. These formulas implicitly partition the gamut and result in specific print attributes. We compared the gamut volume, color constancy, halftone visibility and image graininess for Kueppers' and Demichel's sets of separation formulas. Kueppers' separation formulas enable printing with a slightly larger gamut, but lead to a slightly higher halftone visibility. The image graininess induced by the color separation is a local property and is a result of abrupt changes in color gamut during color separation of neighboring pixels. By minimizing the number of subgamuts that are used for the color separation of a small neighborhood, we can generate smooth metallic-ink images.

In the future, it would be interesting to try new formulations for the color separation that may produce improved quality attributes. The sets of separation formulas that we use in this work are specific to an 8-ink CMYRGBWK system. The approach could be extended to other sets of inks. For artistic or advertisement purposes, we may also combine metallic inks with standard inks to create color images that comprise specularly as well as nonspecularly reflecting image regions.

\section{REFERENCES}

${ }^{1}$ J. Morovič and J. Lammens, "Color management," Colorimetry Understanding the CIE System, edited by J. Schanda (John Wiley, 2007), pp. 159-206.

${ }^{2}$ G. Sharma, "Color fundamentals for digital imaging," Digital Color Imaging Handbook, edited by G. Sharma (CRC Press, 2003), pp. 1-40.
${ }^{3}$ J. Morovič and M. R. Luo, "The fundamentals of gamut mapping: a survey," J. Imaging Sci. Technol. 45, 283-290 (2001).

${ }^{4}$ P. Pjanic and R.D. Hersch, "Specular color imaging on a metallic substrate," Proc. IS\&T's CIC21: Twenty-first Color and Imaging Conf., (IS\&T, Springfield, VA, 2013), pp. 61-68.

${ }^{5}$ V. Babaei and R. D. Hersch, "Juxtaposed color halftoning relying on discrete lines," IEEE Trans. Image Process. 22, 679-686 (2013).

${ }^{6}$ V. Babaei and R. D. Hersch, "Yule-Nielsen based multi-angle reflectance prediction of metallic halftones," Proc. SPIE 9395, 93950H (2015).

7 K. Deshpande, P. Green, and M. R. Pointer, "Gamut evaluation of an $n$-colour printing with the minimum number of measurements," Color Res. Appl. 40, 408-415 (2015).

${ }^{8} \mathrm{H}$. Kueppers, "Printing process where each incremental area is divided into a chromatic area and an achromatic area and wherein the achromatic areas are printed in black and white and the chromatic areas are printed in color sub-sections," US Patent 4,812,899 (1989).

${ }^{9}$ V. Ostromoukhov, "Chromaticity gamut enhancement by heptatone multi-color printing," Proc. SPIE 1909, 139-151 (1993).

${ }^{10} \mathrm{H}$. Boll, "A color to colorant transformation for a seven ink process," Proc. SPIE 2170, 108-118 (1994).

11 D. Tzeng, "Spectral-based color separation algorithm development for multi-ink color reproduction," Ph.D. dissertation (Rochester Institute of Technology, 1999).

12 P. Urban and R. R. Grigat, "Spectral-based color separation using linear regression iteration," Color Res. Appl. 31, 229-238 (2006).

${ }^{13}$ Y. Chen, R. Berns, L. Taplin, and F. Imai, "Multi-ink color separation algorithm improving image quality," J. Imaging Sci. Technol. 52, 020604 (2008).

14 C. H. Son, H. M. Park, and Y. H. Ha, "Improved color separation based on dot-visibility modeling and color mixing rule for six-color printers," J. Imaging Sci. Technol., 55, 010505 (2011).

15 Q. Liu, X. Wan, and D. Xie, "Optimization of spectral printer modeling based on a modified cellular Yule-Nielsen spectral Neugebauer model,"," J. Opt. Soc. Am. A, 31, 1284-1294 (2014).

16 V. Ostromoukhov and R. D. Hersch, "Multi-color and artistic dithering," Proc. SIGGRAPH, in Computer Graphics Proc., Annual Conf. Series (1999), pp. 425-432.

17 J. Morovič, P. Morovič, and J. Arnabat, "HANS: controlling ink-jet print attributes via Neugebauer primary area coverages," IEEE Trans. Image Process. 21, 688-696 (2012).

18 R. Bala, "Device characterization," Digital Color Imaging Handbook, edited by G. Sharma (CRC Press, 2003), pp. 269-382.

19 J. A. S. Viggiano, "Modeling the color of multi-colored halftones," Proc. TAGA 44-62 (1990).

${ }^{20}$ V. Babaei, "Color Reproduction with Juxtaposed Halftoning," Ph.D. thesis 6703, École Polytechnique Fédérale de Lausanne, Lausanne, 2015.

${ }^{21}$ R. Balasubramanian, "Optimization of the spectral Neugebauer model for printer characterization," J. Electron. Imaging 8, 156-166 (1999).

${ }^{22}$ K. Deshpande, P. Green, and M. R. Pointer, "Color separation of an $n$-color printing process using inverse printer models," J. Imaging Sci. Technol. 58, 050504 (2014).

23 G. Sharma, W. Wu, and E. N. Dalal, "The CIEDE2000 color-difference formula," Color Res. Appl. 30, 21-30 (2005). 
${ }^{24}$ R. D. Hersch and A. K. Singla, "An ink spreading model for dot-on-dot spectral prediction," Proc. IS\&T/SID 14th Color Imaging Conf. (IS\&T, Springfield, VA, 2006), pp. 38-43.

${ }^{25}$ M. Hébert and R. D. Hersch, "Review of spectral reflectance models for halftone prints: principles, calibration, and prediction accuracy," Color Res. Appl. 40, 383-397 (2015).

${ }^{26}$ F. Bernardini, J. Mittleman, H. Rushmeier, C. Silva, and G. Taubin, "The ball-pivoting algorithm for surface reconstruction," IEEE Trans. Vis. Comput. Graphics 5, 349-359 (1999).
27 B. Oztan, G. Sharma, and R. P. Loce, "Misregistration sensitivity in clustered-dot color halftones," J. Electron. Imaging 17, 023004 (2008).

${ }^{28}$ M. R. Luo and R. W. G. Hunt, "A chromatic adaptation transform and a colour inconstancy index," Color Res. Appl. 23, 154-158 (1998).

${ }^{29}$ X. Zhang and B. A. Wandell, "A spatial extension of CIELAB for digital color image reproduction," SID Int'l. Symposium, Digest of Technical Papers (1996), pp. 731-734.

${ }^{30}$ R. Ulichney, Digital Halftoning (MIT Press, Cambridge, 1987), pp. 239265. 